\title{
M13 Bacteriophage Functionalized Silver Nanowire Surface-Enhanced Raman Scattering Sensor
}

\author{
Ho Sang Jung ${ }^{1, *}$, Eun Hye Koh ${ }^{1,2}$, Jin-Woo Oh ${ }^{2}$, Sung-Gyu Park ${ }^{1}$, Dong-Ho Kim ${ }^{1, *}$ \\ ${ }^{1}$ Advanced Functional Thin Films Department, Korea Institutes of Materials Science (KIMS), \\ Changwon, Gyeongnam, 51508, Republic of Korea \\ ${ }^{2}$ Department of Nano Fusion Technology, Pusan National University (PNU), Busan, 46241, Republic \\ of Korea \\ jhs0626@kims.re.kr
}

\begin{abstract}
:
A surface-enhanced Raman scattering (SERS) sensor comprising silver nanowires (AgNWs) and genetically engineered M13 Bacteriophages (BPWHW) was fabricated onto a glass-fiber filter paper (GFFP) membrane. AgNWs stacked on GFFP formed high density of SERS-active hot spots and the surface-coated BPWHW functioned as bioreceptor for selective pesticide detection. The Raman signal enhancement and the selective SERS detection of pesticides were investigated compared with wildtype M13 bacteriophage-decorated AgNWs (BPWT/AgNW) and undecorated AgNWs (AgNW). The developed BPWHW/AgNW specifically captured paraquat $(\mathrm{PQ})$ compared with other bipyridylium pesticides such as diquat (DQ) and difenzoquat (DIF). Further, field application test was carried out using hand-held Raman spectrometer on $P Q$ pre-treated apple peels demonstrating the feasibility of paper-based SERS substrate for on-site residual pesticide detection. The developed M13 bacteriophage-functionalized AgNWs SERS sensor is expected to be applied for various pesticides and chemicals detection through modification of the M13 bacteriophage surface peptide sequences.
\end{abstract}

Key words: SERS, M13 bacteriophage, AgNW, pesticides, chemical sensors

\section{Introduction}

Surface-enhanced Raman scattering (SERS) sensors have been widely developed for various molecular detections due to the great Raman signal enhancement characteristic. However, for the selective detection of target molecule, receptors have been utilized such as antibodies, aptamers and macrocycles. Here, as a bio-receptor, M13 bacteriophage was introduced on the SERS sensor surface. M13 bacteriophage is a one-dimensional (1D) virus that expresses 2700 copies of surface proteins. The genetic engineering of surface protein provided enhanced binding affinity toward target molecules. Inspired by 1D wire shape of M13 bacteriophage, network-structured SERS sensor was fabricated together with AgNWs as shown in Figure 1.

\section{Experiments}

M13 bacteriophage solution was mixed with AgNWs solution and vacuum filtration was carried out on GFFP. PQ at various concentrations were dropped on each substrate and dried before Raman measurement. Each Raman measurement condition of exposure time, laser power, and wavelength was fixed as $5 \mathrm{~s}, 6 \mathrm{~mW}$ and $633 \mathrm{~nm}$, respectively. Selectivity test was performed by measuring Raman signal before and after the washing of pesticide containing SERS substrates.

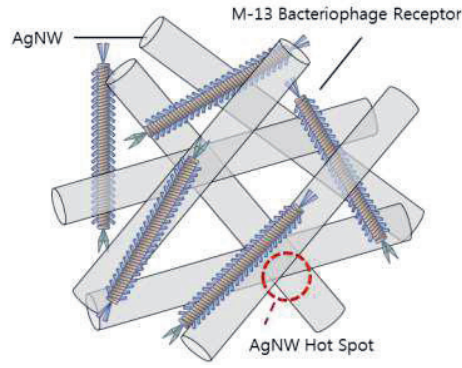

Fig. 1. Schematic illustration of M13 bacteriophage functionalized AgNWs SERS substrate.

\section{Results}

The surface morphology of BPWHW/AgNW was characterized by scanning electron 
microscopy (SEM). As shown in Figure 2a, multi-stacked AgNWs were observed on GFFP. Because the pore size of GFFP was $700 \mathrm{~nm}$ and the length of AgNWs was $20 \mu \mathrm{m}$, it was successfully stacked on GFFP forming network structure that contained a high density of hotspots. The morphology of functionalized M13 bacteriophage was observed by confocal scanning fluorescence microscopy (CSFM). BPWHW was stained with anti-M13 bacteriophage coat protein $g 8 p$ primary antibody followed by red fluorescence dye labeled secondary antibody. As shown in Figure $2 \mathrm{~b}$, the fluorescence signal of M13 bacteriophage was similar with that of AgNWs network structure because the M13 bacteriophages were adhered along the direction of AgNWs. Atomic force microscopy (AFM) and FT-IR analysis were further carried out (data not shown here) and all the materials characterization results confirmed successful functionalization of M13 bacteriophage on AgNWs surface.
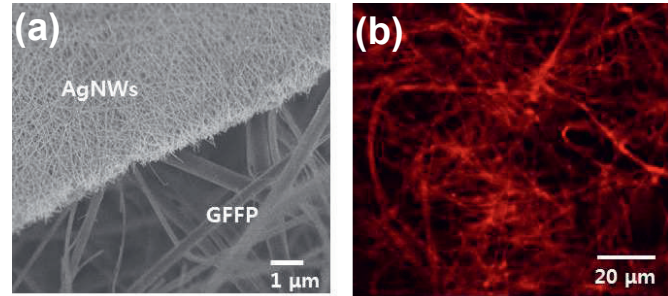

Fig. 2. Surface morphology of BPWHW/AgNW measured by (a) SEM and (b) CSFM.

Then, Raman enhancement of BPWHW/AgNW was tested using $P Q$ molecule at various concentrations. As shown in Figure 3, $P Q$ solutions at initial concentrations in the range of 100 ppm - 1 ppm could be detected at typical $P Q$ Raman peak of $1647 \mathrm{~cm}^{-1}$.

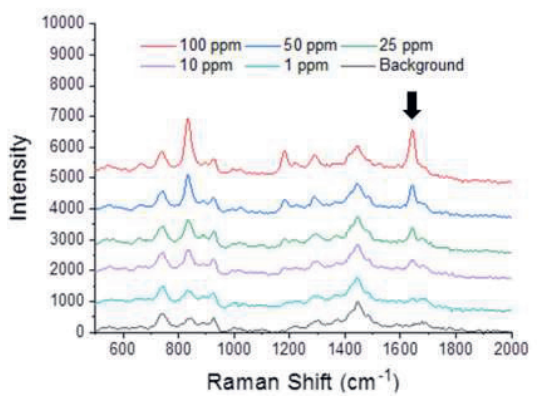

Fig. 3. Raman spectra of $P Q$ at various concentrations.

The selectivity test of PBWHW was performed in the presence of controls such as BPWT/AgNW and bare AgNW substrates. Also, Raman signal was measured before and after the washing step. As shown in Figure 4, PQ on BPWHW/AgNW showed remarkable remaining Raman signal even after the vigorous washing in excess DI water. Further, selectivity of other bipyridiylium pesticides such as DQ and DIF was measured and calculated that capture efficiency of BPWHW/AgNW for each $P Q, D Q$ and DIF molecule was $76.4 \%, 40.4 \%$ and $36.8 \%$, respectively.

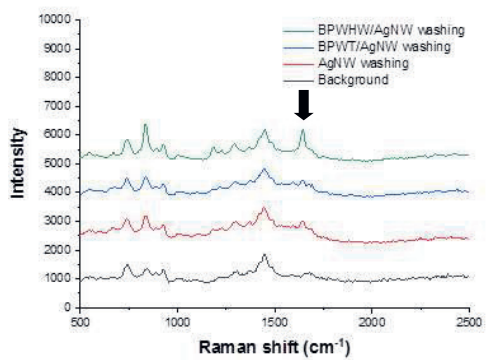

Fig. 4. Raman spectra of $50 \mathrm{ppm} P Q$ solution on $B P W H W / A g N W, B P W T / A g N W$ and AgNW after washing step.

For the field application test, $P Q$ was pretreated on apple surface and dried for $24 \mathrm{~h}$. Then, $P Q$ was transferred to BPWHW/AgNW and measured by hand-held Raman spectrometer. As shown in Figure 5, $\mathrm{PQ}$ concentration as low as $0.1 \mu \mathrm{g} / \mathrm{cm}^{2}$ could be observed.

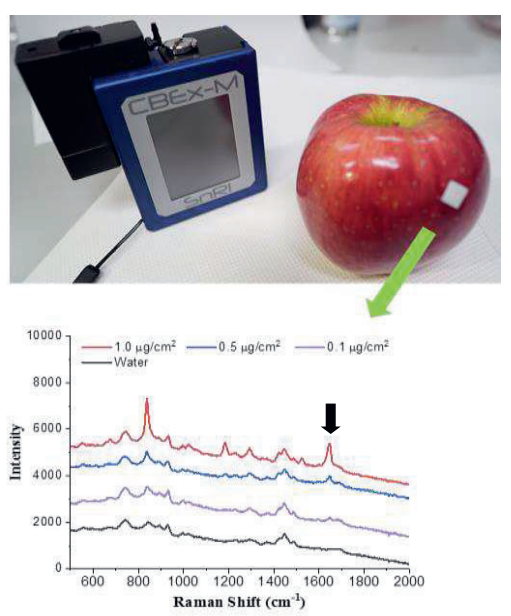

Fig. 5. Photograph of BPWHW/AgNW attached apple surface and detected $P Q$ Raman spectra.

\section{References}

[1] S. -G. Park, C. W. Mun, M. K. Lee, T. Y. Jeon, H. -S. Shim, Y. -J. Lee, J. -D. Kown, C. S. Kim, D. -H. Kim, Adv. Mater. 27, 4290 - 4298 (2015); doi: 10.1002/adma.201501587

[2] J. S. Moon, M. J. Park, W. G. Kim, C. T. Kim, J. Y. Hwang, D. Seol, C. S. Kim, J. R. Sohn, H. Chung, J. W. Oh, Sens. Actuat. B 240, 757-762 (2017); doi: https://doi.org/10.1016/j.snb.2016.09.050 